\title{
Interacting jets from binary protostars
}

\author{
G. C. Murphy ${ }^{1}$, T. Lery ${ }^{2}$, S. O’Sullivan ${ }^{3}$, D. Spicer ${ }^{4}$, F. Bacciotti ${ }^{5}$, and A. Rosen ${ }^{6}$
}

${ }^{1}$ Laboratoire d'Astrophysique de Grenoble, CNRS, Université Joseph Fourier, BP 53, 38041 Grenoble, France e-mail: Gareth.Murphy@obs .ujf-grenoble.fr

2 European Science Foundation, 1 quai Lezay-Marnesia, BP 90015, 67080 Strasbourg, France e-mail: tlery@esf.org

3 UCD School of Mathematical Sciences, University College Dublin, Belfield, Dublin 4, Ireland e-mail: stephen. osullivan@ucd.ie

4 NASA/GSFC Laboratory for Solar and Space Physics, Mail Stop 612.1, Greenbelt, MD 20771, USA e-mail: daniel.s.spicer@nasa.gov

5 INAF - Osservatorio Astrofisico di Arcetri, Largo E. Fermi 5, 50125 Florence, Italy e-mail: fran@arcetri.astro.it

${ }^{6}$ Max-Planck-Institut für Radioastronomie, Auf dem Hügel 69, 53121 Bonn, Germany e-mail: arosen@mpifr-bonn.mpg.de

Received 4 September 2007 / Accepted 13 November 2007

\section{ABSTRACT}

\begin{abstract}
Aims. We investigate potential models that could explain why multiple proto-stellar systems predominantly show single jets. During their formation, stars most frequently produce energetic outflows and jets. However, binary jets have only been observed in a very small number of systems.

Methods. We model numerically 3D binary jets for various outflow parameters. We also model the propagation of jets from a specific source, namely L1551 IRS 5, known to have two jets, using recent observations as constraints for simulations with a new MHD code. We examine their morphology and dynamics, and produce synthetic emission maps.

Results. We find that the two jets interfere up to the stage where one of them is almost destroyed or engulfed into the second one. We are able to reproduce some of the observational features of L1551 such as the bending of the secondary jet.

Conclusions. While the effects of orbital motion are negligible over the jets dynamical timeline, their interaction has significant impact on their morphology. If the jets are not strictly parallel, as in most observed cases, we show that the magnetic field can help the collimation and refocusing of both of the two jets.
\end{abstract}

Key words. ISM: Herbig-Haro objects - ISM: individual objects: LDN 1551 IRS 5 - ISM: jets and outflows - stars: formation

\section{Introduction}

Well-collimated jets occur across a broad range of mass scales from young stellar objects to active galactic nuclei. In young stellar objects (YSOs), jets and outflows are more easily observed at optical, infrared and UV wavelengths and the properties are relatively well known (Reipurth \& Bally 2001; Ray et al. 2007). YSO jets are believed to be launched by means of a magnetic field which is anchored or frozen into a circumstellar disk, and pinched and twisted by the disks rotation. Once the pinched-in field reaches a critical angle, fast-rotating gas from the disk may be loaded onto the field lines and thus launched into the parent cloud in an outflow (Blandford \& Payne 1982). The most accepted schools of thought diverge on whether the wind is launched primarily from the inner disk region (disk wind, Ferreira 1997; Königl \& Pudritz 2000) or the X-annulus where the young star's magnetosphere interacts with the disk (X-wind, Shu et al. 2000). However in either case the jet is believed to brake the rotation of the disk and is thus essential to the star formation process, as it allows the star to accrete up to its final mass.

In all such models a single star with its associated circumstellar disk produces a bipolar outflow or jet. Estimates derived from studies of stellar populations, however, show that large numbers of binary and multiple star systems are expected
(Patience et al. 2002; Simon et al. 1995; Ghez et al. 1993; Duquennoy \& Mayor 1991). Do such systems present associated disks and jets? It has been argued that the disk configuration in a binary system may either be in circumstellar disks or circumbinary disks depending on the star separation distance (Hartigan et al. 2000a). On the other hand, it is well established that multiple sources can be the source of $\mathrm{HH}$ objects, e.g. T Tau, IRAS 04325-1419, Z Cma, Sz 68, SR 24, S Cr A, AS 353 (Reipurth et al. 1993). Now, a multiple system may produce a single jet or outflow or a set of multiple outflows. Although there should be "many" visible binary jets, only a few binary jets from binary protostars have been observed. The examples known to the authors are compiled in Table 1. The frequency of existing binary jets is low compared to the large number of protostellar binary sources.

Observations have also shown that multiple or quadrupolar jets may occur. Reipurth et al. (1993) discovered a second flow HH144 from the same source as HH1-2. Gredel \& Reipurth (1994b) observed a second flow (HH121) from the same source as the well-known HH111 outflow. In both cases there are large angles of separation between the two jets. This can be explained if the disks are not coplanar (Mathieu 1994). However, observations of apparent double jets can also be explained by other means: Avery et al. (1990) imaged the outflow in L723 and found a distinct multi-polar morphology. They concluded that the lobes 
Table 1. Evidence of binary jets and outflows?

\begin{tabular}{lllll}
\hline \hline Outflow (source) & Natal cloud & RA (J2000) & Dec (J2000) & Reference \\
\hline HH154 (L1551 IRS 5) & Tau-Aur & 043134.20 & +180804.8 & Liseau et al. (2005) \\
HH1-2-HH144 & Orion & 053622.85 & -064606.6 & Reipurth et al. (1993) \\
HH111-HH121 & Orion & 055146.07 & +024830.6 & Gredel \& Reipurth (1994a) \\
L723 (IRAS 19156+1906) & Cep & 191753.16 & +191216.6 & Anglada (2004) \\
HH288 (IRAS 00342+6347) & Cep & 003711.07 & +640359.8 & Gueth et al. (2001) \\
HH377 (IRAS 23011+6126) & Cep E & 230313.9 & +614221 & Ladd \& Hodapp (1997) \\
IRAS 16293-2422 & $\rho$ Oph E & 163222.8 & -242833 D & Hirano et al. (2001) \\
IRAS 20050+2720 & Cyg Rift & 200706.7 & +272853 & Bachiller et al. (1995) \\
\hline
\end{tabular}

of the jets were in fact shell walls of two cavities swept clear by a single bipolar outflow. Anglada et al. (1991) discovered a double radio source at the centre of the outflow structure and this led to a reappraisal of the of the double outflow theory (Anglada et al. 1996, 1998; Girart et al. 1997; Hirano et al. 1998; Palacios \& Eiroa 1999; Shirley et al. 2002; Estalella et al. 2003; Anglada 2004). Other example of multiple molecular outflows include IRAS 16293-2422, IRAS 20050+2720.

In order to explore these possibilities, and to investigate about the nature of jets from multiple systems, we perform numerical simulations of binary jets with typical physical quantities. Our aim is to demonstrate the effects of their relative sizes and speeds on their interaction, in order to test if the jet evolution can lead to situations compatible with the few binary jets observed, and with the corresponding observed properties.

As a starting point, we focus on jets emanated independently by two sources that launch the two outflows with a small angle of separation. A suitable test case for this scenario is the the binary jet HH 154, emanating from the source L1551 IRS 5.

Numerical models of optical jets have evolved over the past twenty years, from the original models by Norman et al. (1981) of the Blandford \& Rees (1974) twin-exhaust model, to later models by Hartmann \& Raymond (1984); Hartigan et al. (1987); Raymond et al. (1988) which were $1.5 \mathrm{D}$ bow shock models which explicitly tried to model observed features, to axisymmetric and 3D geometries and including such physics as atomic radiative cooling (Blondin et al. 1990; Raga \& Noriega-Crespo 1998; Stone \& Hardee 2000; Raga et al. 2000, 2001).

In our case we model a fully three-dimensional binary jet system using ATLAS, a new shock-capturing, multidimensional, constrained transport, adaptive-grid, directionally unsplit, higher-order Godunov astrophysical MHD code, ATLAS and examine its morphology and propagation dynamics.

The paper is structured as follows: in Sect. 2 we describe the observed properties of the binary jet L1551 IRS 5, that constitutes our test case. In Sect. 3 we describe the methodology, the set of equations used, the microphysics included, the numerical code and the initial and boundary conditions on the computational grid. In Sect. 4 we present the results of the simulation, and finally in Sect. 5 we discuss our results and the insights brought forward by our model.

\section{Observations of jets from the binary protostar L1551 IRS 5}

The picture being built up over 25 years of observations of the object L1551 IRS 5 and its associated jets and outflows is that of a pair of YSOs each with its own associated disk and outflow, the whole structure embedded in a larger disk.

Strom et al. (1976) observed the near-infrared source IRS 5 within the Lynds molecular cloud 1551 (L1551). The source has an infrared emission nebulosity (Hodapp 1994), consistent with the presence of a channel perpendicular to the high-density disk, from which the light from the central star escapes and irradiates the nebulosity. Rodríguez et al. (1998) confirmed that L1551 IRS 5 was a binary system and show the first images of the circumbinary disks and the red lobes of the two jets. Additionally they suggested that there is a circumbinary structure and a largescale envelope around L1551 IRS 5. Lay et al. (1994) observed a large circumbinary disk of $\sim 160 \mathrm{AU}$ in diameter. Rodríguez et al. (2003) confirmed the jet binarity at radio wavelengths, while Liseau et al. (2005) provided a value for binary separation of $40 \mathrm{AU}$. We add that recent observational evidence has suggested that the source may have a small third companion (Lim \& Takakuwa 2006).

Cudworth \& Herbig (1979) discerned two fast Herbig-Haro objects near the IRS 5 source. Snell et al. (1980) saw for the first time the "remarkable double-lobed structure" in L1551. This was the among the first molecular bipolar outflows discovered. Bieging \& Cohen (1985) identified IRS 5 as a binary source. Moriarty-Schieven \& Wannier (1991) and Pound \& Bally (1991) identified a second outflow from the same source. Mundt et al. (1991) also observed two independent rows of knots although these were interpreted as edges of a limb-brightened cavity, and were only identified as jets by Fridlund \& Liseau (1998) (hereafter FL), who measured a jet angular separation of approximately $20^{\circ}$.

Recent observations carried out with the Hubble Space Telescope and SUBARU have further clarified the morphology of the flow (Fridlund et al. 2005). The optical jets extend southwest and disappear at approximately 1400 AU from the IRS5. The north and south jets appear to be launched from the south and north disks respectively. According to Liseau et al. (2005) the northern jet is faster with a radial velocity projected in the plane of the sky of $\sim 430 \mathrm{~km} \mathrm{~s}^{-1}$ whereas the southern jet has a radial velocity at most $65 \mathrm{~km} \mathrm{~s}^{-1}$. Itoh et al. (2000) observed a twisted morphology in the light of iron lines, and argued that the precession of the sources is too slow to be responsible for this effect. Possible other mechanisms can be magnetic in nature e.g. the Lorentz forces (Fendt \& Zinnecker 1998, 2000) or the bending may be caused by the decrease in kinetic energy allowing the ambient magnetic pressure to be comparable to the ram pressure of the jet. Indeed, Scarrott (1988) observed a high degree of polarization in the optical light emitted by the region and concluded that it could be explained by a toroidal field in the cloud around the outflows. Lucas \& Roche (1997) also observed this "peculiar pattern of alignment". The physical cause for the polarimetry pattern remains unexplained.

For completeness we mention also that X-ray emission from the region of the head of the north jet was observed by Favata et al. (2002). Bally et al. (2003) performed a higher angular resolution study, finding a source of X-rays in L1551 IRS 5 at the 


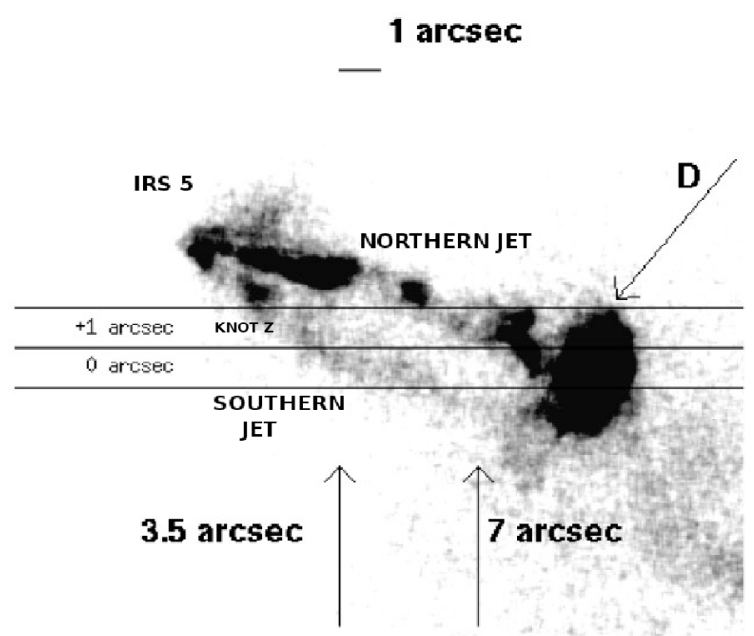

Fig. 1. Fridlund \& Liseau (1998) provide a HST R-band image of the two jets from the binary protostar L1551 IRS 5, located in the constellation Taurus. (Image courtesy of Fridlund \& Liseau 1998.)

location of the source which they attributed to either fast shocks or reflected X-rays from IRS 5 scattered out through the outflow cavity. Bally et al. (2003) put forward a number of models to explain the X-ray emission, including the intriguing possibility of a quasi-stationary X-ray luminous shock maintained by interacting colliding winds between the two protostars.

\section{Methodology}

Our main goal is to model binary jets. Contrary to single jet propagation, that can be modelled also analytically in an axisymmetric approximation, the propagation and interaction of binary jets is an intrinsically three-dimensional problem. A numerical study then becomes necessary, and requires the use of a fully three-dimensional time-dependent model with magnetic field and radiative cooling. Constraints on current computational capacity require us to make a number of approximations however, that lead to the adoption of an ideal magneto-hydrodynamics (MHD) approach. In this way we are able to include the effects of the magnetic field without losing the advantages of using a simple macroscopic description for a fluid particle. We additionally include the non-ideal physics of optically thin atomic radiative cooling losses. Moreover, in our scheme the ionisation fraction of hydrogen is followed in the simulation. This is important as several studies (Bacciotti \& Eislöffel 1999; Podio et al. 2006) have demonstrated that this quantity in jets decouples from the thermal gas conditions and follows the evolution of gas recombination.

\subsection{System of equations}

The ideal MHD equations evolve in time the four quantities (two vector and two scalar) which are conserved in a volume: density $\rho$, momentum, $\rho \boldsymbol{u}$, magnetic flux density $\boldsymbol{B}$ and energy density, $E$.

$\frac{\partial \rho}{\partial t}+\nabla \cdot(\rho \boldsymbol{u})=0$

$\frac{\partial}{\partial t}(\rho \boldsymbol{u})+\nabla \cdot\left[\rho \boldsymbol{u} \otimes \boldsymbol{u}+\left(p^{*}\right) \overline{\overline{\boldsymbol{I}}}+\boldsymbol{B} \otimes \boldsymbol{B}\right]=0$

$\frac{\partial \boldsymbol{B}}{\partial t}+\nabla \cdot(\boldsymbol{u} \otimes \boldsymbol{B}-\boldsymbol{B} \otimes \boldsymbol{u})=0$

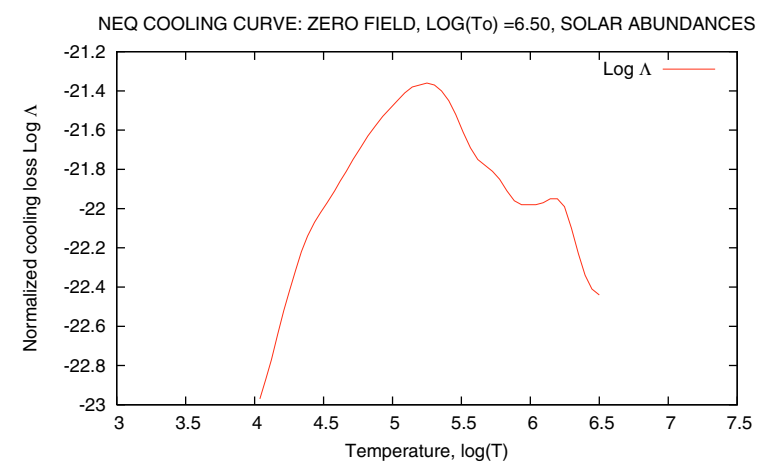

Fig. 2. Log normalised radiative cooling loss plotted against log temperature (Sutherland \& Dopita 1993).

$\frac{\partial E}{\partial t}+\nabla \cdot\left[\left(E+p^{*}\right) \boldsymbol{u}-(\boldsymbol{u} \cdot \boldsymbol{B}) \boldsymbol{B}\right]+L_{\text {cooling }}=0$

where $E$ is the total energy, arising from the sum of three terms, namely kinetic, internal and magnetic energy:

$E=\frac{1}{2} \rho|\boldsymbol{u}|^{2}+\frac{p}{\gamma-1}+\frac{1}{2}|\boldsymbol{B}|^{2}$

and $p^{*}$ is the total (thermal plus magnetic) pressure:

$p^{*}=p+\frac{1}{2}|\boldsymbol{B}|^{2}$

$L_{\text {cooling }}$ represents the losses due to optically thin radiative cooling, and is described in the next paragraph. The units are chosen so that $\boldsymbol{B}$ absorbs a factor of $1 / \sqrt{4 \pi}$. The adiabatic index is $\gamma=5 / 3$ for a monatomic gas throughout the simulations. The equation of state is the ideal gas equation $(p=n k T)$.

\subsection{Microphysics}

Protostellar jets are strongly cooled by radiation of collisionally excited lines, many of which are optically thin. It it this emission which render the objects visible, particularly in $\mathrm{H} \alpha$ and in the light of forbidden lines like the doublet of singly ionised sulphur at about $6700 \AA$ ([SII] $\lambda \lambda 6716,6731)$. Modelling electron transitions directly, however, is neither feasible nor desirable in the fluid approximation. Therefore, we represent the microphysics of energy lost due to radiative cooling using a cooling function adapted from Sutherland \& Dopita (1993) and depicted in Fig. 2, which represents on a macroscopic scale the rate of energy loss at our temperature range for a sample (solar) set of abundances. Sutherland \& Dopita (1993) include the effects of electron collisional ionisation, radiative and dielectronic recombination and line radiation within their cooling function.

To calculate the energy loss in the gas one also needs to know the free electron density, which is computed explicitly at each time step and location in the fluid, as in Falle \& Raga (1995). As mentioned above, this is necessary because the ionisation fraction of hydrogen is not in equilibrium with the thermal properties of the gas, due to the fact that at the observed densities the recombination times are long with respect to the crossing time of the visible portion of the jet. For a thorough discussion of this point see Raga et al. (1997); Bacciotti \& Eislöffel (1999).

The cooling function shown in Fig. 2 is highly non-linear. If we use the cooling timestep to evolve the overall jet the process will be extremely time-consuming. Therefore we separate the cooling from the dynamical evolution at each timestep, first calculating the dynamical change and then computing the radiative cooling using a short substep. 
Table 2. Observational data for L1551 IRS 5 outflows.

\begin{tabular}{ll}
\hline \hline N Jet radial velocity & $200-250 \mathrm{~km} \mathrm{~s}^{-1 a}$ \\
S Jet radial velocity & $65 \mathrm{~km} \mathrm{~s}^{-1 b}$ \\
Binary separation & $45 \mathrm{AU}^{c}$ \\
Angle between Jets & $20^{\circ d}$ \\
Orbital period & $255 \mathrm{years}^{e}$ \\
Ambient density & $5000 \mathrm{~cm}^{-3 f}$ \\
Jet density & $500 \mathrm{~cm}^{-3 g}$ \\
Jet length & $1400 \mathrm{AU}^{h}$ \\
\hline
\end{tabular}

${ }^{a}$ Hartigan et al. (2000b); ${ }^{b}$ Hartigan et al. (2000b); ${ }^{c}$ Liseau et al. (2005); ${ }^{d}$ Fridlund \& Liseau (1998); ${ }^{e}$ Itoh et al. (2000); ${ }^{f}$ Fridlund \& Liseau (1998); ${ }^{g}$ Fridlund \& Liseau (1998); ${ }^{h}$ Fridlund \& Liseau (1998).

\subsection{The numerical code}

We computed the three-dimensional simulations using ATLAS, a new modular, parallel, shock-capturing, directionally unsplit, adaptive-grid, multi-dimensional, constrained transport, higherorder Godunov astrophysical MHD code. Validation and verification tests, including but not limited to the Orszag-Tang MHD vortex, the Brio-Wu and Ryu-Jones suite of shock tube tests and $2 \mathrm{D}$ and $3 \mathrm{D}$ blast wave tests, have been run against the code to build confidence in its ability to form correct solutions. ATLAS uses the PARAMESH (MacNeice et al. 2000) hierarchical block-structured adaptive mesh refinement for high effective resolution in areas of physical interest. The solenoidal constraint $(\nabla \cdot \boldsymbol{B}=0)$ is preserved using a staggered mesh algorithm based on the Balsara \& Spicer (1999) field transport method. ATLAS uses a Piecewise Parabolic scheme (Colella \& Woodward 1984) to reconstruct the values at cell interfaces and the Roe-Balsara approximate MHD Riemann solver (Roe 1981; Roe \& Balsara 1996) to explicitly compute the cell interfaces fluxes. The multidimensional correction used to compute the transverse fluxes is the Corner Transport Upwind scheme of Colella (1990) as modified by Saltzman (1994). In our own case, the simulations were carried out using ATLAS on a 64 node cluster of the so-called "Beowulf" type.

\subsection{Initial and boundary conditions}

Consistently with the location of the object in Taurus, we assumed a distance of 140 parsecs to the jets. We used values derived from the observations for the density, temperature and velocities, as described in Table 2 . The ambient medium is modelled with a uniform density $\left(n_{\mathrm{a}}=5 \times 10^{3} \mathrm{~cm}^{-3}\right)$ and temperature $\left(10^{2} \mathrm{~K}\right)$ and the jets are modelled with density $n_{\mathrm{j}}=0.1 n_{\mathrm{a}}$, temperature $10^{4} \mathrm{~K}$ and velocities of 100 and $300 \mathrm{~km} \mathrm{~s}^{-1}$ respectively (Liseau et al. 2005). Regarding the flow velocity, we take the radial velocities of Hartigan et al. (2000b) $200 \mathrm{~km} \mathrm{~s}^{-1}$ and $60 \mathrm{~km} \mathrm{~s}^{-1}$ and deproject them from the plane of the sky assuming (with Hartigan et al. 2000b) an inclination angle of 45 degrees. In order to reproduce the knotty structure of the flows we also use a sinusoidally varying injection velocity (Raga et al. 1990), assuming an amplitude of $\pm 30 \%$ in the velocity with a period of 8 years for each jet. We also stagger the launching of the two jets, arguing that the faster northern jet is launched $150 \mathrm{yr}$ after the slower southern jet. The calculations of the launch times are based on estimating the age of the jets from their lengths and current velocities, assuming the velocities have not changed over time. The velocity profile across the jet section is a positive cosine - with its maximum at $v_{\text {jet }}$ and its minimum (zero) at $r_{\text {jet }}$. This profile is chosen based on the high angular resolution observations of the DG Tau jet by Bacciotti et al. (2000), where the
Table 3. Initial conditions for simulation.

\begin{tabular}{lr}
\hline \hline Domain & $-840 \mathrm{AU}<y, z<840 \mathrm{AU}$ \\
& $0<x<1680 \mathrm{AU}$ \\
Refinement & 5 levels \\
Max Resolution & $2.625 \mathrm{AU}$ \\
N Jet deprojected velocity & $v_{j}=300 \mathrm{~km} \mathrm{~s}^{-1}$ \\
S Jet deprojected velocity & $v_{j}=100 \mathrm{~km} \mathrm{~s}^{-1}$ \\
Jet radius & $r_{\mathrm{j}}=10^{14} \mathrm{~cm}^{-3}$ \\
Jet density & $n_{\mathrm{j}}=500 \mathrm{~cm}^{-3}$ \\
Ambient density & $n_{\mathrm{a}}=5000 \mathrm{~cm}^{-3}$ \\
Jet Temperature & $T_{\mathrm{j}}=10^{4} \mathrm{~K}$ \\
Ambient Temp & $T_{\mathrm{a}}=100 \mathrm{~K}$ \\
Angle between jets & $0($ Case I \& III) \\
Angle between jets & 10 (Case II) \\
\hline
\end{tabular}

highest velocities appear to be found in the axial region of the jet.

Typical postshock cooling lengths estimated using the plane parallel shock models of Hartigan et al. (1987) are of the order of $30 \mathrm{AU}$ in the colliding outer wings of the binary jet bow shocks and in the internal shocks so will be resolved in $\sim 12$ points.

The initial jets' diameters are approximately 13 points each and the jet cross-sectional areas are resolved in approximately 150 square cells for each jet.

The boundary conditions are inflow for $r<r_{\mathrm{j}}$, reflecting for $r>r_{\mathrm{j}}$ along the inner $x$-boundary $(x=0)$ and outflow along all other boundaries.

\section{Results}

We present here results from fully 3D HD and MHD simulations of the jets.

\subsection{Case I: Hydrodynamic case}

By using a simple hydrodynamical (HD) model, we were able to reproduce the twisted morphology of the L1551 IRS 5 outflow, that in this case appears to arise from the interaction of the two jets. In the observations of L1551 IRS 5, the two jets emerge from a binary source separated by $45 \mathrm{AU}$. The observed angular separation is 20 degrees which corresponds to a deprojected angle of approximately 10 degrees. Initially, for the hydrodynamic case we assume the jets are parallel. (In the next Sect. (4.2) we remove this simplifying assumption.) We model the jet propagation and show that the interaction affects the propagation.

The density slice from the 3D HD simulation (see Fig. 3) clearly shows that the slow, secondary jet appears to bend close to the inlet. Such a bend or kink is also observed in the slow, southern jet of L1551 IRS 5 about 4" (560 AU at a distance of 140 pc from the source (Itoh et al. 2000)). In the simulation shown in Fig. 3 a kink in the slow southern jet is also visible - both in the density midplane cut shown in Fig. 3 and in the derived emission maps shown in 7 . This is caused by the bow shock of the fast jet interacting with the beam of the slow jet. The northern jet has a Mach number $~ 3$ times higher than the slow southern jet and simply pushes it out of the way. This reproduces the observed kink at 4 " - without the need for magnetic fields. There is no noticeable reaction by the fast jet - possibly indicating that the estimated velocity is too high. 


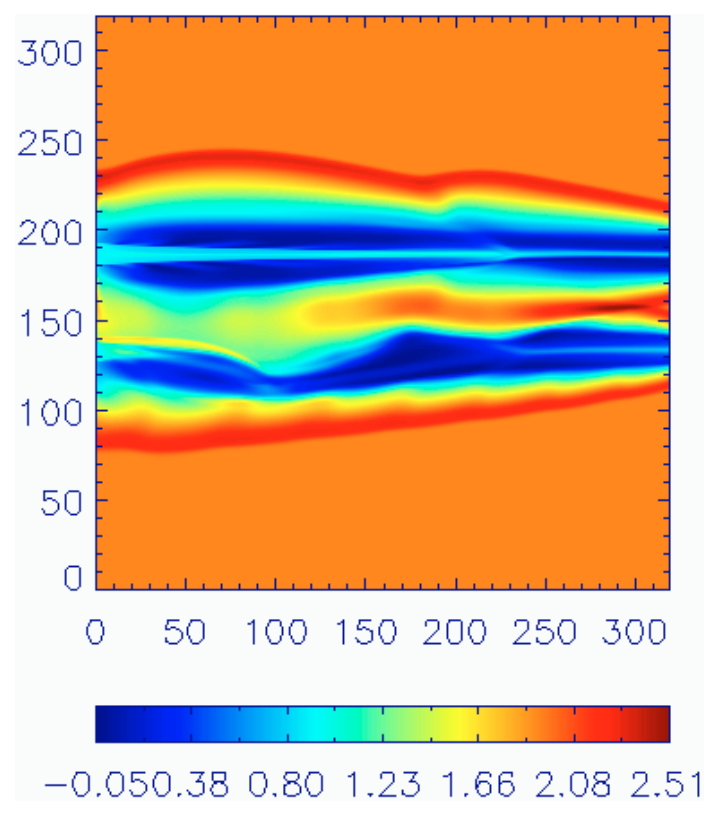

Fig. 3. Midplane slice colour density map of 3D binary jet simulation at $t=190$ years. The scale of the grid is 1680 AU by 1680 AU.

\subsection{Case II: Magneto-hydrodynamic case}

In the second case, we consider the inclusion of the magnetic field and run a full magneto-hydrodynamic (MHD) simulation, trying to reproduce the jet behaviour closer to the source. The magnetic field provides a mechanism for the southern jet to change its direction - hence we allow the jets to initially diverge. The projected angle between the two jets is about 20 degrees (Fridlund \& Liseau 1998) we use a deprojected angle of $10^{\circ}$. To redirect the two jets would require either a density contrast e.g. the wall of a conveniently shaped cavity or a magnetic hoop stress (Bally et al. 2003).

We use the magnetic field configuration based on the observations of Scarrott (1988), that suggest the presence of a toroidal magnetic field around the system. The toroidal field may be produced by the circumbinary disk twisting the frozen-in local magnetic field lines (see, e.g. Matt \& Pudritz 2004, and the references therein.)

We assume that the ambient medium is permeated by a toroidal field with a maximum value of $B=10 \mu \mathrm{G}$. The magnetic vector potential $\boldsymbol{A}$, where $\boldsymbol{B}=\nabla \times \boldsymbol{A}$, had the analytical form $A_{y}=\cos 2 \pi x+\cos 2 \pi y$. This is an approximation to the real field which would be helical according to the disk-twisting theory.

Figure 4 shows a series of density slices from the 3D MHD simulation. The binary outflow is modelled in full 3D with toroidal magnetic field. Compared to the previous HD simulation, we have set the angle between the two jets to be close to $10^{\circ}$. In the hydrodynamic case there is no mechanism to to force the jets to become parallel. In the MHD case the Lorentz force can redirect both of the jets. As a consequence, we would expect to see the two jets propagating into two different directions on large scales. On the contrary, the smaller jet is refocused along the faster jet. The secondary jet (i.e., the south low velocity one), bends towards the $z$-axis, impelled by Lorentz force. This can be seen very clearly in Fig. 4, panel 1 for instance, where the slow jet is already moving towards the centre before the approach of the fast jet. Eventually, the jet material from the slower jet gets merged into the faster one.
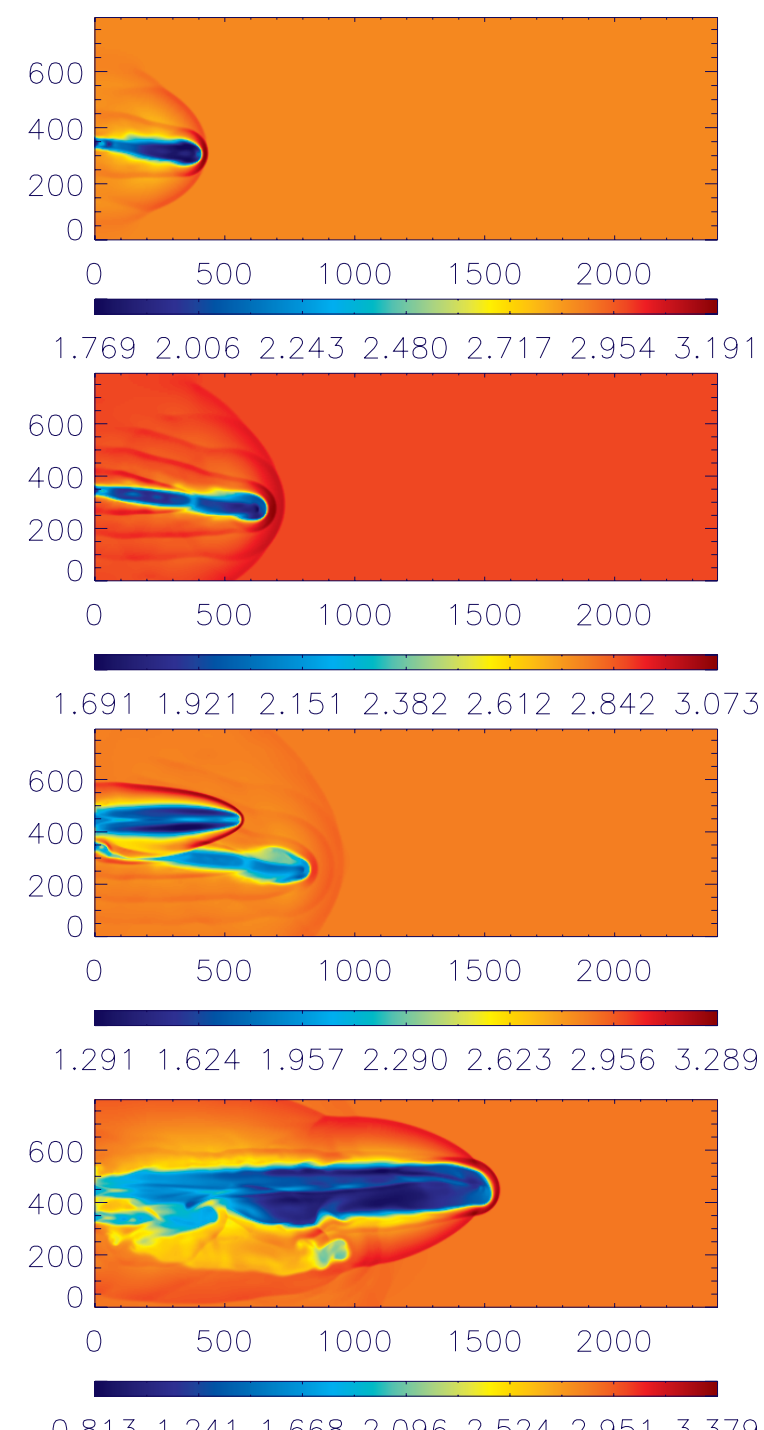

Fig. 4. Time evolution of $\log$ jet total density in the MHD binary jets at $t=42.5,85,127.5,170 \mathrm{yr}$. The images shown are 2D midplane cuts through the $3 \mathrm{D}$ grid.

The observations of L1551 show both jets diverging from the source until about 4 " from the source when they change direction to pursue a roughly parallel course. The change in direction is most pronounced for the southern jet which has the lower ram pressure and thus is easier to redirect. In the simulation over the time evolution we see the southern jet change its direction to move parallel to the axis of the toroidal field. Hence, it appears that the hoop stress from the jet toroidal field slowly changes the direction of the jets. Thus, magnetised binary jets will tend to collimate and refocus along the direction of the fastest or strongest jet.

\subsection{Case III: Orbiting binary jet}

We now explore the effects of orbital motion of the jet sources on the survival of the binary jets. Masciadri \& Raga (2002) have modelled jets from orbiting sources However they modelled just a single jet orbiting a central body and compared its evolution to an analytical prediction. Figure 6 shows a model of a binary jet from two sources orbiting a common centre of mass. The jet crosses the grid in $90 \mathrm{yr}$. In this case, for simplicity we assume 

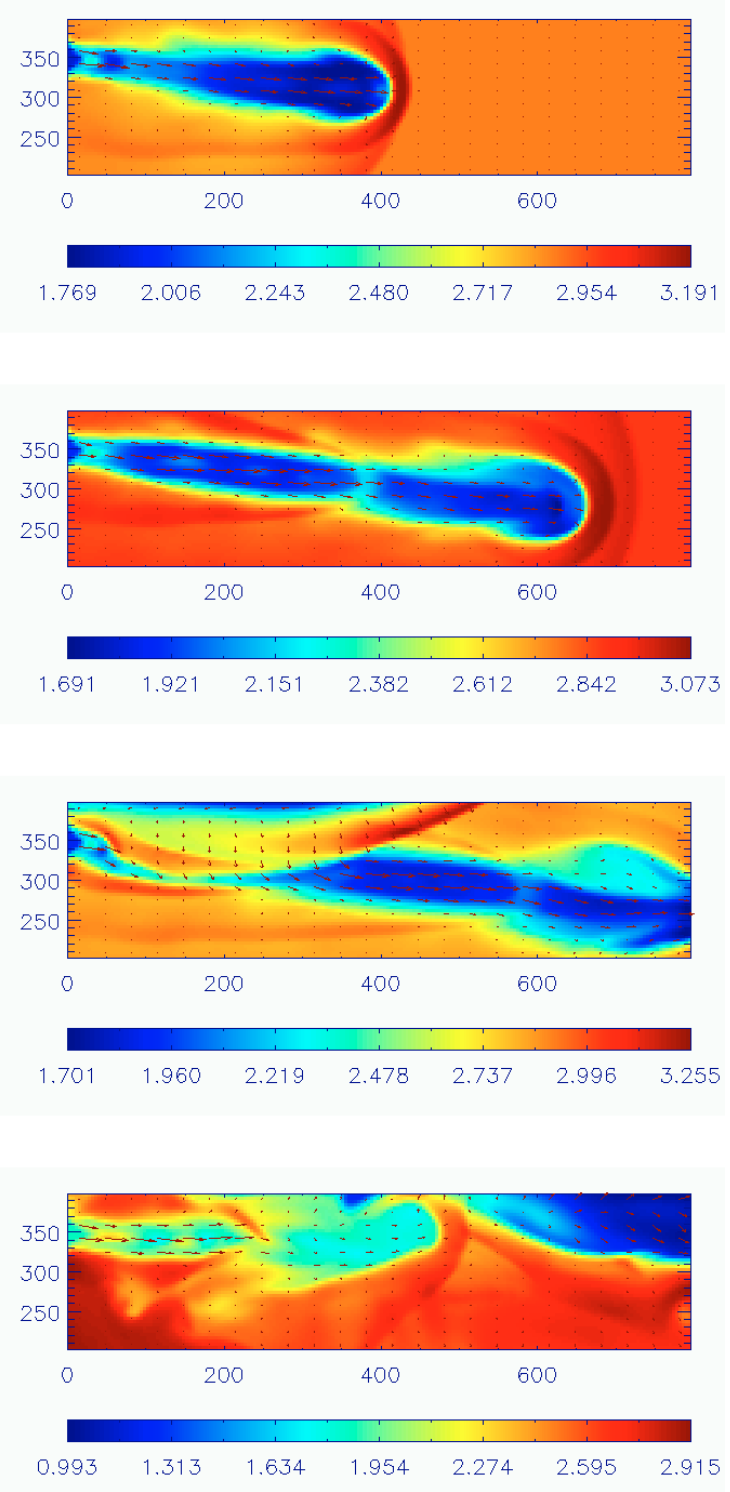

Fig. 5. Section of Fig. 4 showing velocity vectors.

that the jets are launched at the same time and their velocities are 300 and $100 \mathrm{~km} \mathrm{~s}^{-1}$.

We find that, by calculating the dynamical age of the jets in L1551 IRS 5, the orbital period of the binary $\sim 260 \mathrm{yr}$ is too long to have much effect on the fast northern jet (crossing time $\sim 90 \mathrm{yr}$ ).

\subsection{Emission maps}

In order to properly compare the results of our simulations with the existing observations we have computed synthetic emission maps in the light of the forbidden lines of neutral oxygen ([OI] $\lambda 6300)$. In order to predict the line emission produced by the two jets in our model we use the density, temperature and the fraction of ionised hydrogen as computed by the numerical simulation, to find the emissivity in each point of the jet gas. The emissivity $\epsilon$ is defined as the energy emitted at the wavelength $\lambda$

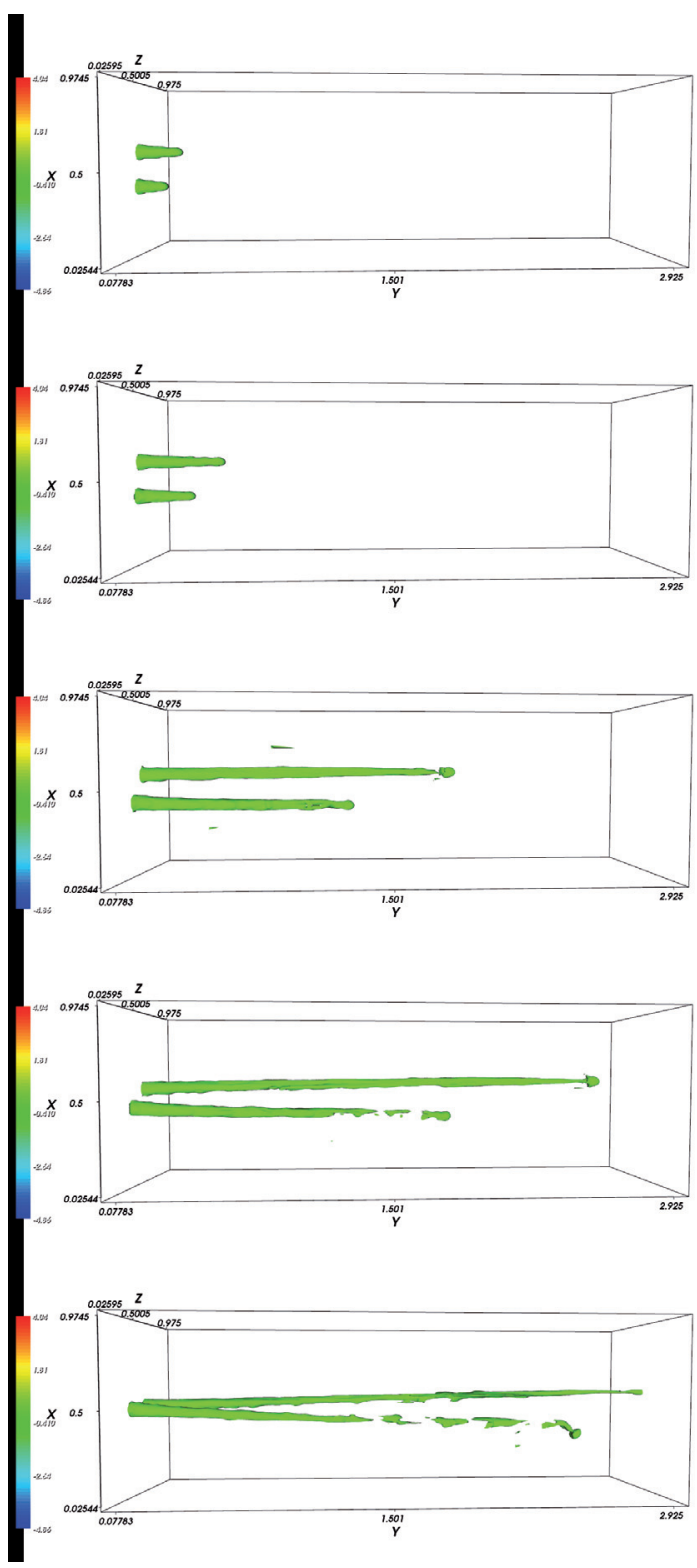

Fig. 6. Surface plot of density of 3D binary jet simulation at $t=$ $5,10,40,60,90 \mathrm{yr}$. The orbital period of the source is 260 years. The jet pair has performed $1 / 3$ of a rotation.

of the transition by a unit volume of gas per unit time. Following Bacciotti (2002), the emissivity can be expressed as:

$\epsilon_{Z^{i}, \lambda}=A_{\lambda} \frac{h c}{\lambda} x_{\mathrm{e}} n_{\mathrm{H}}^{2}\left(\frac{Z^{i}}{Z}\right)\left(\frac{Z}{H}\right)\left(\frac{n_{\text {upper }}}{n\left(Z^{i}\right)}\right)$

where $A_{\lambda}$ is the transition probability, $n_{\mathrm{H}}$ is the hydrogen nuclear number density, $x_{\mathrm{e}}$ is the ionised fraction of hydrogen, $\frac{Z^{i}}{Z}$ is the fraction of the element $Z$ ionised at level $i, \frac{Z}{H}$ is the abundance of element $Z$ with respect to hydrogen, and $\frac{n_{\text {upper }}}{n\left(Z^{i}\right)}$ is the fractional population of the upper level of the transition. We assume solar abundances for the elements in the fluid. For O, following Bacciotti \& Eislöffel (1999), we can determine the fractional ionisation as a function of $x_{\mathrm{e}}$ assuming the relationship

$\frac{\mathrm{O}^{+}}{\mathrm{O}^{0}}=\frac{\left(\mathrm{C}_{\mathrm{O}}+\delta_{\mathrm{O}}\right) x_{\mathrm{e}}}{\left(\alpha_{\mathrm{O}}-\delta_{\mathrm{O}}\right) x_{\mathrm{e}}+\delta_{\mathrm{O}}^{\prime}}$ 


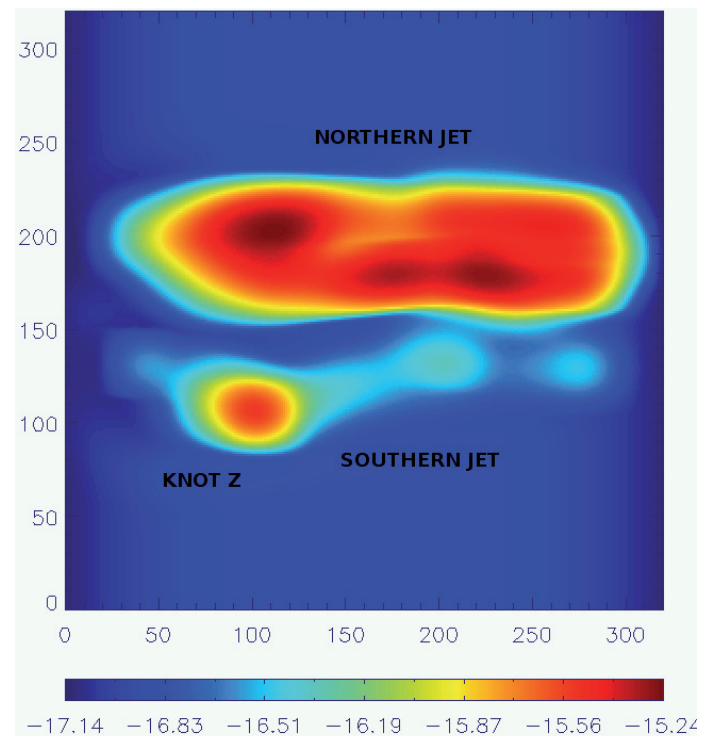

Fig. 7. Synthetic [OI] $\lambda 6300$ emission map derived from the physical parameters of our simulation in the hydrodynamic case as depicted in Fig. 3. to be compared with corresponding HST observations of the L1551 IRS 5 jet (HH154) (see text) The image shows the northern and southern jets at a time $t=190 \mathrm{yr}$. Knot Z indicates the point where the bow shock of the northern jet collides with the beam of the southern jet. The units are erg cm${ }^{-2} \mathrm{~s}^{-1} \operatorname{arcsec}^{-1}$.

holds, where $C_{\mathrm{O}}$ is the collisional ionization rate, $\alpha_{\mathrm{O}}$ the direct plus dielectronic recombination rates, $\delta_{\mathrm{O}}$ and $\delta_{\mathrm{O}}^{\prime}$ are the direct and inverse charge ionisation exchange rates respectively. The fractional population of the upper level, $\frac{n_{\text {upper }}}{n\left(Z^{i}\right)}$, is basically a function of the free electron density and the electron temperature, and is determined by a 5-level linear system of equations describing the statistical equilibrium of the level populations subject to collisional excitation and de-excitation, and to spontaneous radiative decay. A more complete description of the method can be found in Bacciotti et al. (1995); Bacciotti \& Eislöffel (1999) and Bacciotti (2002).

The emissivity values calculated in the $3 \mathrm{D}$ space from our simulation are then integrated along lines of sight, assuming an inclination angle of 45 degrees. The result is then convolved with the beam of the instrument, which corresponds to an angular resolution of $0^{\prime} 1$ in the HST case.

The synthetic emission map produced in this way is shown in Fig. 7. It is clear that the interaction has a strong observational signature. The second jet is virtually obliterated but remains visible at the point where the fast jet's bow shock impinges on its beam. The emission map is quite different to what is seen in the density slice. The knotty structure of the southern jet and the asymmetric structure of the northern jet are only apparent in the emission map. These may be compared with the observations in Fig. 1. Even though the image has been convolved to account for the beam resolution a peak in emission is visible in the southern jet where the bow shock of the fast jet is striking the beam. In the observations a similar peak in the emission is visible near the source of the southern jet (Knot Z in Fig. 1).

\section{Discussion}

The L1551 IRS 5 jet pair can be seen as a pathological object unlike any other observed at such close range. In fact many effects, like binary source orbiting, peculiar environment, density parameters, magnetic fields play a role in sculpting this most unusual object. To attempt an explanation of the observed phenomena that can take into account all the above mentioned elements, a full 3D numerical treatment is necessary. In this paper we have presented 3D HD and MHD simulations of interacting binary jets that may help explain the L1551 IRS 5 case. We have used the code ATLAS including radiative cooling and nonequilibrium ionisation treatment. The main results can be summarised as follows:

- We can reproduce the kink and bending of the secondary jet and the knotty structure of the two components as observed for L1551 IRS 5.

- Over a long time-scale, we see precession induced by the orbital motion of the source. On the short lifetime of the jets from L1551, this effect is negligible.

- If the jets are not strictly parallel, as in most observed cases, we show that the magnetic field can help the collimation and refocusing of both the jets. In fact the toroidal field affects the motion of the southern jet; and this magnetically driven change in direction together with the interaction of the bow shock of the fast jet with the beam of the slow jet contribute to the distinctive morphology.

- We have produced emission maps in the [OI] lines which can be compared with observations. We show that the kink structure of interacting jets is still apparent in the synthetic observations.

Some aspects however remain to be clarified. For example, one of them is the nature of the the source of the X-ray emission. Bally et al. (2003) find a source of X-rays in L1551 IRS 5 which they attribute to either fast shocks or reflected X-rays scattered out through the outflow cavity. The fast shocks may be caused by jet collimation which could be magnetic in nature. In our model there is a peak in emission (Knot Z in Fig. 1) which appears to come from the colliding winds. The Bally model suggests that the X-rays come from a moving source at the base of the jets. If the density ratio is $n_{\text {ambient }} / n_{\text {jet }}=10$ then the shock speed will be too slow to achieve X-ray emission temperatures. If on the other hand the density ratio is lower i.e. 0.1 the jet is moving into a less dense region swept out by the slower jet the shock velocities will be higher. To get up to the observed shock velocity the density contrast would need to be much lower than observed e.g. 0.01 would give a velocity in the range required. We also could assume that it is caused by magnetic reconnection in the interval between the two jets where the field is compressed by the pair of bow shocks.

One other interesting aspect to be discussed is the implications for jet launching of the binarity of the source. The binary provides a vastly different natal environment for a protostar and its attendant jets and outflows than that envisaged in a single star formation. We identify three main differences for jet launching for binaries, orbital motion, circumbinary material and interaction with a jet. In this work we showed that the impact upon the jet beam of the bow shock of its neighbour is large enough to be observable as a knot of emission moving along along the jet. The orbital motion is of too long a period to affect the jet propagation. The role of the circumbinary material may be to twist up the magnetic field into the toroidal shape which appears in the polarized light. In conclusion, our simulations have demonstrated to be able to reproduce the observed signatures in this source and to give powerful constraints on the physical effects at play. A larger number of observed cases has however to be modeled with our code in order to give a complete view of binary jet production. 
Acknowledgements. This work was carried out as part of the CosmoGrid project, funded under the Programme for Research in Third Level Institutions (PRTLI) administered by the Irish Higher Education Authority under the National Development Plan and with partial support from the European Regional Development Fund. The PARAMESH software used in this work was developed at the NASA Goddard Space Flight Center under the HPCC and ESTO/CT projects. Some of this work was carried out using ICHEC/Cosmogrid computers and under the HPC-EUROPA project (RII3-CT-2003-506079), with the support of the European Community Research Infrastructure Action under the FP6 Structuring the European Research Area Programme). The authors acknowledge support through the Marie Curie Research Training Network JETSET (Jet Simulations, Experiments and Theory) under contract MRTN-CT-2004-005592. The authors wish to acknowledge the SFI/HEA Irish Centre for High-End Computing (ICHEC) for the provision of computational facilities and support.

\section{References}

Anglada, G. 2004, in Rev. Mex. Astron. Astrofis. Conf. Ser., 100 Anglada, G., Estalella, R., Rodriguez, L. F., et al. 1991, ApJ, 376, 615 Anglada, G., Rodriguez, L. F., \& Torrelles, J. M. 1996, ApJ, 473, L123 Anglada, G., Rodriguez, L. F., \& Torrelles, J. M. 1998, in Star Formation with the Infrared Space Observatory, ASP Conf. Ser., 132, 303

Avery, L. W., Hayashi, S. S., \& White, G. J. 1990, ApJ, 357, 524

Bacciotti, F. 2002, in Rev. Mex. Astron. Astrofis. Conf. Ser., 8

Bacciotti, F., \& Eislöffel, J. 1999, A\&A, 342, 717

Bacciotti, F., Chiuderi, C., \& Oliva, E. 1995, A\&A, 296, 185

Bacciotti, F., Mundt, R., Ray, T. P., et al. 2000, ApJ, 537, L49

Bachiller, R., Fuente, A., \& Tafalla, M. 1995, ApJ, 445, L51

Bally, J., Feigelson, E., \& Reipurth, B. 2003, ApJ, 584, 843

Balsara, D. S., \& Spicer, D. S. 1999, J. Comput. Phys., 149, 270

Bieging, J. H., \& Cohen, M. 1985, ApJ, 289, L5

Blandford, R. D., \& Payne, D. G. 1982, MNRAS, 199, 883

Blandford, R. D., \& Rees, M. J. 1974, MNRAS, 169, 395

Blondin, J. M., Fryxell, B. A., \& Königl, A. 1990, ApJ, 360, 370

Colella, P. 1990, J. Comput. Phys., 87, 171

Colella, P., \& Woodward, P. 1984, J. Comput. Phys., 54, 174

Cudworth, K. M., \& Herbig, G. 1979, AJ, 84, 548

Duquennoy, A., \& Mayor, M. 1991, A\&A, 248, 485

Estalella, R., Palau, A., Girart, J. M., et al. 2003, in Rev. Mex. Astron. Astrofis. Conf. Ser., 135

Falle, S. A. E. G., \& Raga, A. C. 1995, MNRAS, 272, 785

Favata, F., Fridlund, C. V. M., Micela, G., Sciortino, S., \& Kaas, A. A. 2002 , A\&A, 386, 204

Fendt, C., \& Zinnecker, H. 1998, A\&A, 334, 750

Fendt, C., \& Zinnecker, H. 2000, in IAU Symp., 112

Ferreira, J. 1997, A\&A, 319, 340

Fridlund, C. V. M., \& Liseau, R. 1998, ApJ, 499, L75

Fridlund, C. V. M., Liseau, R., Djupvik, A. A., et al. 2005, A\&A, 436, 983

Ghez, A. M., Neugebauer, G., \& Matthews, K. 1993, ApJ, 106, 2005

Girart, J. M., Estalella, R., Anglada, G., et al. 1997, ApJ, 489, 734

Gredel, R., \& Reipurth, B. 1994a, A\&A, 289, L19

Gredel, R., \& Reipurth, B. 1994b, A\&A, 289, L19

Gueth, F., Schilke, P., \& McCaughrean, M. J. 2001, A\&A, 375, 1018
Hartmann, L., \& Raymond, J. C. 1984, ApJ, 276, 560

Hartigan, P., Raymond, J., \& Hartmann, L. 1987, ApJ, 316, 323

Hartigan, P., Bally, J., Reipurth, B., \& Morse, J. A. 2000a, Protostars and Planets IV, 841

Hartigan, P., Morse, J., Palunas, P., Bally, J., \& Devine, D. 2000b, AJ, 119, 1872 Hirano, N., Hayashi, S. S., Umemoto, T., \& Ukita, N. 1998, ApJ, 504, 334

Hirano, N., Mikami, H., Umemoto, T., Yamamoto, S., \& Taniguchi, Y. 2001, ApJ, 547, 899

Hodapp, K.-W. 1994, ApJS, 94, 615

Itoh, Y., Kaifu, N., Hayashi, M., et al. 2000, PASJ, 52, 81

Königl, A., \& Pudritz, R. E. 2000, Protostars and Planets IV, 759

Ladd, E. F., \& Hodapp, K.-W. 1997, ApJ, 474, 749

Lay, O. P., Carlstrom, J. E., Hills, R. E., \& Phillips, T. G. 1994, ApJ, 434, L75

Lim, J., \& Takakuwa, S. 2006, ApJ, 653, 425

Liseau, R., Fridlund, C. V. M., \& Larsson, B. 2005, ApJ

Lucas, P. W., \& Roche, P. F. 1997, MNRAS, 286, 895

MacNeice, P., Olson, K., Mobarry, C., de Fainchtein, R., \& Packer, C. 2000, Computer Phys. Comm., 126, 330

Masciadri, E., \& Raga, A. C. 2002, ApJ, 568, 733

Mathieu, R. D. 1994, ARA\&A, 32, 465

Matt, S., \& Pudritz, R. E. 2004, ApJ, 607, L43

Moriarty-Schieven, G. H., \& Wannier, P. G. 1991, ApJ, 373, L23

Mundt, R., Ray, T. P., \& Raga, A. C. 1991, A\&A, 252, 740

Norman, M. L., Smarr, L., Smith, M. D., \& Wilson, J. R. 1981, ApJ, 247, 52

Palacios, J., \& Eiroa, C. 1999, A\&A, 346, 233

Patience, J., White, R. J., Ghez, A. M., et al. 2002, ApJ, 581, 654

Podio, L., Bacciotti, F., Nisini, B., et al. 2006, A\&A, 456, 189

Pound, M. W., \& Bally, J. 1991, ApJ, 383, 705

Raga, A., \& Noriega-Crespo, A. 1998, AJ, 116, 2943

Raga, A. C., Binette, L., Canto, J., \& Calvet, N. 1990, ApJ, 364, 601

Raga, A. C., Mellema, G., \& Lundqvist, P. 1997, ApJS, 109, 517

Raga, A. C., Curiel, S., Rodríguez, L. F., \& Cantó, J. 2000, A\&A, 364, 763

Raga, A., Cabrit, S., Dougados, C., \& Lavalley, C. 2001, A\&A, 367, 959

Ray, T., Dougados, C., Bacciotti, F., Eislöffel, J., \& Chrysostomou, A. 2007, in Protostars and Planets V, ed. B. Reipurth, D. Jewitt, \& K. Keil (Tucson: University of Arizona Press), 951, 231

Raymond, J. C., Hartmann, L., \& Hartigan, P. 1988, ApJ, 326, 323

Reipurth, B., \& Bally, J. 2001, ARA\&A, 39, 403

Reipurth, B., Heathcote, S., Roth, M., Noriega-Crespo, A., \& Raga, A. C. 1993, ApJ, 408, L49

Rodríguez, L. F., D’Alessio, P., Wilner, D. J., et al. 1998, Nature, 395, 355

Rodríguez, L. F., Porras, A., Claussen, M. J., et al. 2003, ApJ, 586, L137

Roe, P. L. 1981, J. Comput. Phys., 43, 357

Roe, P. L., \& Balsara, D. S. 1996, SIAM J. Appl. Math., 56, 57

Saltzman, J. 1994, J. Comput. Phys., 115, 153

Scarrott, S. M. 1988, MNRAS, 231, 39P

Shirley, Y. L., Evans, N. J., \& Rawlings, J. M. C. 2002, ApJ, 575, 337

Shu, F. H., Najita, J. R., Shang, H., \& Li, Z.-Y. 2000, Protostars and Planets IV, 789

Simon, M., Ghez, A. M., Leinert, C., et al. 1995, ApJ, 443, 625

Snell, R. L., Loren, R. B., \& Plambeck, R. L. 1980, ApJ, 239, L17

Stone, J. M., \& Hardee, P. E. 2000, ApJ, 540, 192

Strom, S. E., Vrba, F. J., \& Strom, K. M. 1976, AJ, 81, 638

Sutherland, R. S., \& Dopita, M. A. 1993, ApJ, 88, 253 